\title{
Nanobarrier-Terminated Growth of Single-Walled Carbon Nanotubes on Quartz Surfaces
}

\author{
Chaoqun Feng, Yagang Yao, Jin Zhang $(\bowtie)$, and Zhongfan Liu $(\bowtie)$ \\ Beijing National Laboratory for Molecular Sciences (BNLMS), Key Laboratory for the Physics and Chemistry of Nanodevices, \\ State Key Laboratory for Structural Chemistry of Unstable and Stable Species, College of Chemistry and Molecular Engineering, \\ Peking University, Beijing 100871, China
}

Received: 15 April 2009 / Revised: 6 May 2009 / Accepted: 6 May 2009

(C) Tsinghua University Press and Springer-Verlag 2009. This article is published with open access at Springerlink.com

\begin{abstract}
Using carbon nanotubes as nanobarriers, the growth of single-walled carbon nanotubes (SWNTs) on a quartz surface can be terminated. First, carbon nanotube nanobarriers were grown on a quartz surface by the gas flowdirected growth mode. Then, the SWNTs were grown on the quartz surface via the lattice-oriented growth mode, in which growth of SWNTs can be terminated by hitting the nanotube nanobarriers. Moreover, using the carbon nanotube nanobarrier as a marker, the mechanism of the growth of SWNTs on the quartz surface can be studied; a base-growth mechanism is indicated. Based on this termination process and the base-growth mechanism, SWNT arrays with controlled lengths can be grown on a quartz surface by fixing the sites of both catalysts and nanobarriers.
\end{abstract}

\section{KEYWORDS}

Single-walled carbon nanotubes, terminated growth, carbon nanotube nanobarrier, length control of carbon nanotubes

\section{Introduction}

Since the discovery of single-walled carbon nanotubes (SWNTs) in 1993 [1], significant progress has been made in their synthesis and application in a wide variety of fields. However, controlled synthesis of SWNTs, especially in respect of their diameter and chirality, is still a challenge. To make progress in this area, further understanding of the growth mechanisms is required. Generally, there are two growth modes for SWNTs: tip-growth and basegrowth. The growth mechanism for both modes is the same, i.e., the vapor-liquid-solid (VLS) growth mechanism [2] in which the carbon species dissolve into the catalyst and precipitate out to form carbon nanotubes. Based on the VLS mechanism, many methods have been developed to grow SWNTs in a controlled manner. For example, the size distribution of catalyst nanoparticles can strongly influence the distribution of nanotube diameters [3, 4]; special catalysts can grow SWNTs with narrow chirality distributions [5, 6]; site-controlled catalyst nanoparticles determine the growth position of carbon nanotubes [7]; the carbon feeding rate during the growth can be used to control the diameter distribution of SWNTs [8]; the growth of SWNTs

Address correspondence to Jin Zhang, jinzhang@pku.edu.cn; Zhongfan Liu, zfliu@pku.edu.cn 
floating in the gas phase $[9,10]$ can afford ultra-long carbon nanotubes (up to centimeters in length); and on single-crystal substrates, such as sapphire [11, 12] and quartz [13, 14], SWNTs with extremely high alignment can be grown. However, for an individual carbon nanotube, it is difficult to control its growth, because the active growth time and growth rate depend on the activity of the catalyst, which is uncontrollable.

We report herein a rational approach to terminate the growth of SWNTs on a quartz surface by means of carbon nanotube nanobarriers. First, carbon nanotube nanobarriers were grown on a quartz surface by the gas flow-directed growth mode. Then the SWNTs were grown on the quartz surface via the lattice-oriented growth mode, during which SWNT growth can be terminated by the carbon nanotube nanobarriers. Moreover, using the carbon nanotube nanobarrier as a marker, experimental indication of a base-growth mechanism for SWNT growth on the quartz surface can be obtained. Based on this termination and base-growth mechanism, SWNT arrays with controlled lengths can be grown on a quartz surface by fixing the sites of both catalysts and nanobarriers.

\section{Experimental}

\subsection{Preparation of ultra-long carbon nanotubes (nanobarriers)}

As the substrate, we used a single crystal stable temperature (ST)-cut quartz wafer which was annealed for $8 \mathrm{~h}$ at $900{ }^{\circ} \mathrm{C}$ in air before chemical vapor deposition (CVD) growth of SWNTs. First, the nanobarriers-ultra-long carbon nanotubes, perpendicular to the [100] crystallographic direction-were synthesized on the quartz substrate by the gas flow-directed growth mode [15]. $\mathrm{FeCl}_{3}$ was used as the catalyst and methane was used as the feed gas. The catalyst lines were patterned by polydimethylsiloxane (PDMS)-stamping a solution of $\mathrm{FeCl}_{3}$ in ethanol and were only patterned near one edge of the substrate and parallel to the [100] crystallographic direction. After reducing the catalyst in $\mathrm{H}_{2}$ for $10 \mathrm{~min}$ at $980{ }^{\circ} \mathrm{C}, \mathrm{CH}_{4}(8 \mathrm{sccm})$ and $\mathrm{H}_{2}(16$ $\mathrm{sccm})(\mathrm{sccm}=$ standard cubic centimeters per minute) gases were introduced at the same temperature for 30 min. Then, $\mathrm{CH}_{4}$ was replaced by $\mathrm{Ar}$ and the furnace was cooled to room temperature.

\subsection{Growth of SWNTs on the surface modified} with the nanobarriers

Lattice-oriented SWNTs were grown by thermal CVD of ethanol on substrates modified with nanobarriers (ultra-long carbon nanotubes). Colloidal $\mathrm{Fe}_{2} \mathrm{O}_{3}$ in ethanol (Fe concentration of $3 \mathrm{mmol} / \mathrm{L}$ ), was spincoated onto the substrate at $2000 \mathrm{rpm}$ for $1 \mathrm{~min}$. After reduction by $\mathrm{H}_{2}\left(50 \mathrm{sccm}\right.$ ) for $10 \mathrm{~min}$ at $900{ }^{\circ} \mathrm{C}$, ethanol was introduced via an argon bubbler $(15 \mathrm{sccm})$ for $15 \mathrm{~min}$. Then, the furnace was cooled down to room temperature in $\mathrm{Ar}$ and $\mathrm{H}_{2}$. SWNTs were also grown on the same quartz substrate at $925{ }^{\circ} \mathrm{C}$ to illustrate the influence of temperature.

\subsection{Characterization of SWNTs and AFM manipulation of the nanobarriers}

Scanning electron microscopy (SEM, Hitachi S-4700, operated at $1 \mathrm{kV}$ accelerating voltage) and atomic force microscopy (AFM, DI Nanoscope III, operated in tapping mode) were used to characterize the SWNTs. AFM manipulation of the nanobarriers was carried out along a predefined path parallel to the lattice-oriented carbon nanotubes from the meeting point with the SWNTs, using homemade software and procedures [16].

\section{Results and discussion}

Figure $1(\mathrm{a})$ is a schematic illustration of the termination of SWNT growth on a quartz surface using carbon nanotubes as nanobarriers. The nanobarrier nanotubes were first grown on stable temperature (ST)-cut quartz substrates through the gas flow-directed growth mode with the gas flow perpendicular to the [100] crystallographic direction. Then SWNTs were grown along the lattice direction at a lower temperature. Different from the gas flow-directed nanotubes, the orientations of the later SWNTs were found to align along the [100] crystallographic direction. When the lattice-oriented SWNTs met the nanobarrier nanotubes, the growth 
of the lattice-oriented SWNTs was terminated by the nanobarriers. The ultra-long nanometer-height nanotubes acted as physical walls which were difficult for the lattice-oriented SWNTs to cross over. A similar phenomenon has been found by the Rogers's group [17] who used a patterned layer of amorphous $\mathrm{SiO}_{2}$ (2 $\mathrm{nm}$ thickness) deposited by electron beam evaporation; some of the SWNTs stopped growing at the interface of the $\mathrm{SiO}_{2}$ pattern because of the surface relief. Figures 1(b) and 1(c) are typical SEM images of SWNTs terminated by nanotube nanobarriers. Most of the lattice-oriented SWNTs stopped growing just at the meeting point with the ultra-long SWNTs (nanobarriers). Because of the low activity of some catalyst sites, some of the carbon nanotubes stopped growing before they met the nanobarriers.
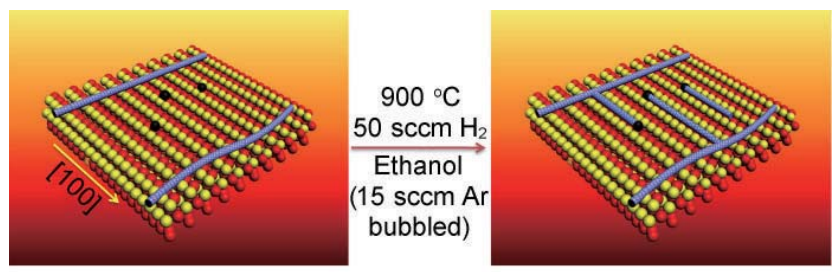

(a)

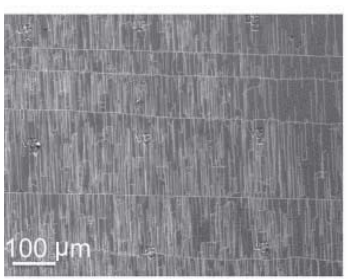

(b)

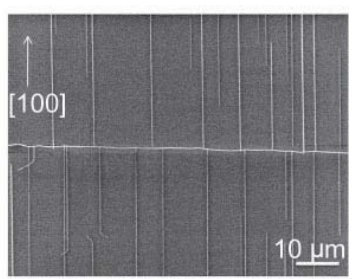

(c)
Figure 1 (a) Schematic illustration of the experimental approach: the nanobarrier carbon nanotubes are synthesized first, and then the nanobarriers terminate the growth of lattice-oriented carbon nanotubes. The red and yellow balls correspond to oxygen atoms and silicon atoms, respectively. The lattice direction and reaction condition is also shown. (b), (c) SEM images of typical results under different magnification

The detailed structure at the meeting points of SWNTs and nanobarriers was characterized by AFM. Figure 2 shows AFM images of four different types of meeting points observed in our experiments. In Fig. 2(a), the lattice-oriented SWNT was stopped just at the meeting point with the nanobarrier nanotube. In Fig. 2(b), the lattice-oriented SWNT hit the nanobarrier nanotube and the force from the growth of the lattice-oriented SWNT actually stretched the nanobarrier nanotube. In Fig. 2(c), the lattice-oriented
SWNT hit and stretched the nanobarrier nanotube and then, to a greater extent than that in Fig. 2(b), went beyond the nanobarrier nanotube. In Fig. 2(d), the lattice-oriented SWNTs simply crossed over the nanobarrier nanotube without stretching it.

Further analysis indicates that the structures appearing in Fig. 2 are dependent on the diameters of the lattice-oriented SWNTs and the nanobarrier nanotubes. In Figs. 2(a), 2(b), 2(c), and 2(d), the nanobarrier nanotube is the same nanotube with a diameter of $3.0 \mathrm{~nm}$, while the lattice-oriented SWNTs have a diameter of 1.3, 1.1, 1.4, and $3.2 \mathrm{~nm}$, respectively. When the diameter of the lattice-oriented SWNT is significantly smaller than the diameter of the nanobarrier nanotube, the growth can be easily terminated, even when a lattice-oriented carbon nanotube goes beyond this nanobarrier. Because the carbon nanotube can only grow through slit between the nanobarrier and the quartz surface, the friction from both sides makes the growth terminate soon after the barrier. AFM manipulation showed that the lattice-oriented SWNT was on the silica surface under the nanobarrier nanotube: while manipulating

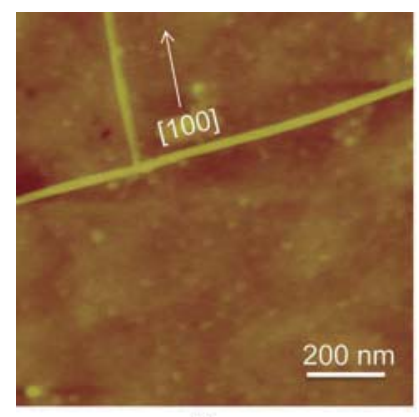

(a)

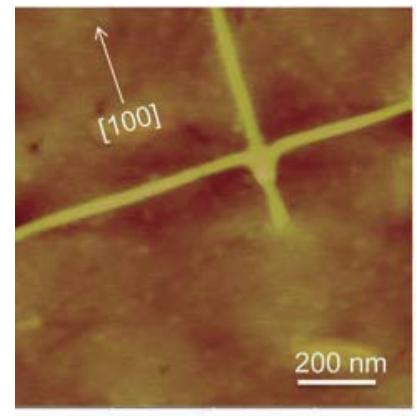

(c)

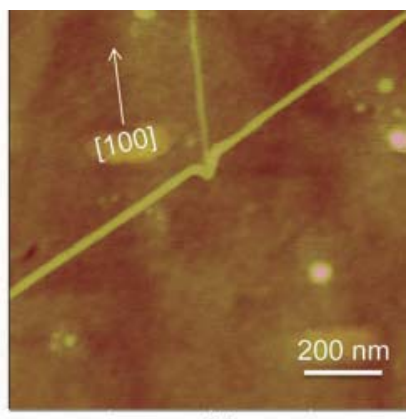

(b)

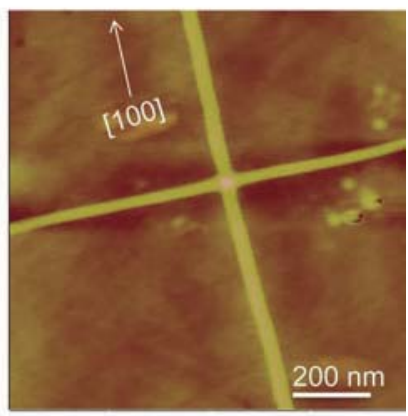

(d)
Figure 2 AFM images of different structures at the meeting points of the lattice-oriented SWNT and the nanobarrier nanotube. From (a) to (d), the diameter of the lattice-oriented SWNTs is 1.3, 1.1, 1.4, and $3.2 \mathrm{~nm}$, respectively. The diameter of the nanobarrier nanotube is 3 $\mathrm{nm}$ in each case 
the nanobarrier, there was no obvious movement of the latticeoriented carbon nanotube. But when the diameter of the latticeoriented SWNT is close to, or larger than, the diameter of the nanobarrier nanotube, there is the possibility of the latticeoriented SWNT crossing over the barrier and keeping growing as shown in Fig. 2(d). This

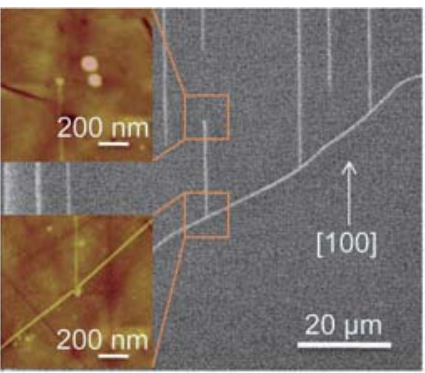

(a)

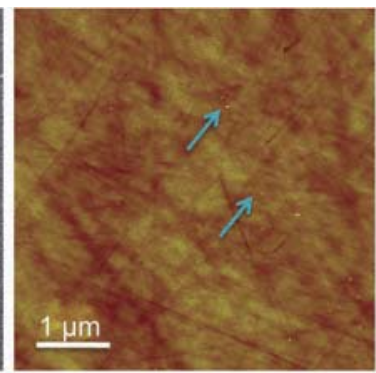

(b)

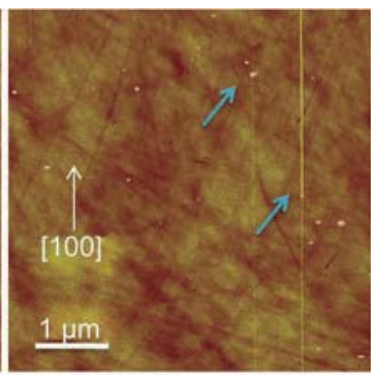

(c)

Figure 3 (a) SEM image of lattice-oriented SWNTs terminated by a gas flow-directed carbon nanotube. The insets are AFM images of the two ends of the lattice-oriented carbon nanotube. (b), (c) AFM images at the same position before and after growth of lattice-oriented SWNTS

Interestingly, a "sickle" shaped structure [19] was observed in some lattice-oriented SWNTs, especially when the reaction temperature was increased from $900{ }^{\circ} \mathrm{C}$ (shown in Fig. 4(a)) to $925{ }^{\circ} \mathrm{C}$ (shown in Fig. $4(\mathrm{~b}))$. The reason may be that SWNTs are more likely to flow along the gas direction at higher temperature. SWNTs with a "sickle" structure could be formed by a multi-step mechanism as follows. First, the nanotubes grow standing up from the catalyst, and once the nanotubes have grown to a considerable length, the ends of the nanotubes without the catalyst

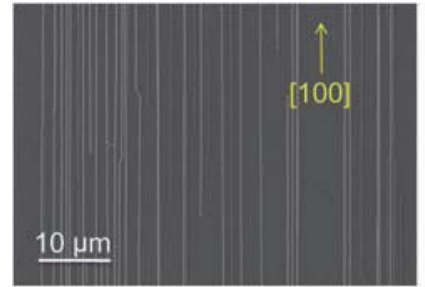

(a)

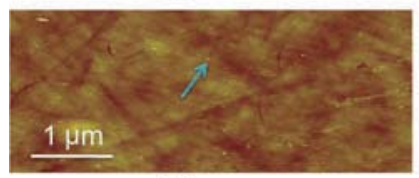

(c)

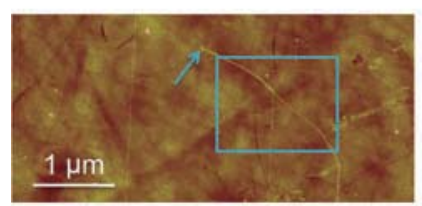

(e)

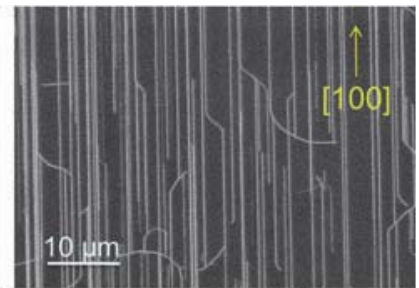

(b)

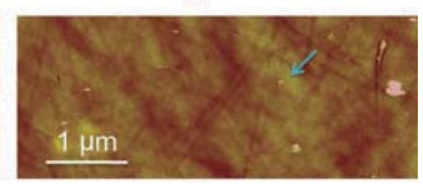

(d)

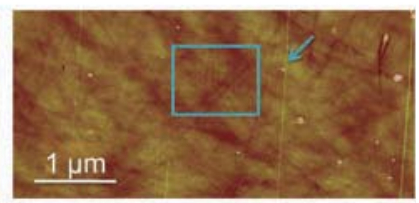

(f) short carbon nanotubes as well as in long SWNTs ( $>1$ $\mathrm{mm})$. Although we cannot exclude the existence of tip-growth, we think that base-growth predominates. Furthermore, the alignment can be attributed to strong anisotropic van der Waals interactions between SWNTs and the single crystal quartz surface [20].
Figure 4 SEM image of lattice-oriented carbon nanotubes grown at different temperatures: (a) $900{ }^{\circ} \mathrm{C}$, (b): $925^{\circ} \mathrm{C}$., AFM images before (c) and after (e) the growth of lattice-oriented carbon nanotubes at 925 ${ }^{\circ} \mathrm{C}$, with a 'sickle' shaped structure visible at the end near the catalyst in (e). AFM images before (d) and after ( $f$ ) the growth of latticeoriented carbon nanotubes at $925^{\circ} \mathrm{C}$, with self-organized nanotube serpentines visible at the end near the catalyst in ( $f$ ) 
drop down to the substrate. Then the driving force of base-growth makes the carbon nanotubes go ahead along the lattice direction until termination, and finally the section of the SWNTs near to the catalyst falls onto the surface to form a "sickle" shaped structure (shown in Fig. 4(e)) or self-organized carbon nanotube serpentines [17, 21] (shown in Fig. 4(f)). The position of the catalyst did not change during the growth process, which means that the base-growth mechanism is also consistent with the "sickle" shaped SWNTs growth at higher temperature. Our results clearly reveal the importance of the temperature and provide a viable way to obtain aligned nanotube arrays with fewer "sickle" shaped structures by lowering the reaction temperature.

Based on the termination phenomenon, SWNT arrays with controlled length can be grown by controlling the relative position of the catalyst particles and the nanobarrier nanotubes. The lengths of lattice-oriented SWNTs are dictated by the distance between the catalyst particles and the nanobarriers. The catalyst lines were patterned by a needle lithography method [22] in which an iron needle tip coated with $\mathrm{FeCl}_{3}$ solution was used to define catalyst patterns on quartz. The alignments of the catalyst lines and nanobarrier nanotubes were carried out under an optical microscope. Figure 5 shows SEM

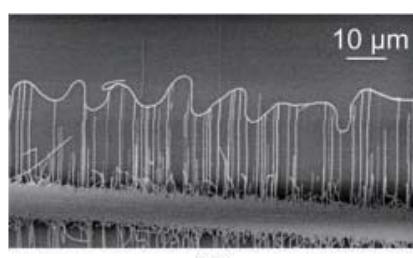

(a)

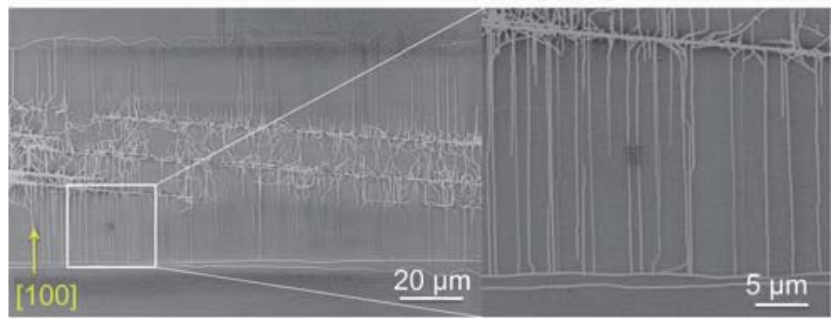

(c)

Figure 5 (a), (b) SEM images of the SWNTs with different lengths grown from the catalyst pattern terminated by the curved nanobarriers; (c) SEM images of a representative result under different magnification. The aligned SWNTs grown from the catalyst pattern extend to both sides of the pattern, until they are terminated by the nanobarriers images of lattice-oriented SWNTs with controlled lengths obtained by controlling the distance between the catalyst lines and the nanobarrier nanotubes. When lattice-oriented SWNTs growing from the same catalyst line were terminated by the same nanobarrier nanotube, which was parallel to the catalyst line, the lengths of the lattice-oriented SWNTs were nearly uniform. In contrast, if the nanobarrier is a curved carbon nanotube, the SWNTs grown from the same catalyst pattern will have different lengths.

\section{Conclusions}

Gas flow-directed ultra-long SWNTs can be used as nanobarriers to terminate the growth of latticeoriented SWNTs on a quartz surface. This method is very simple and involves no damage to the substrate. Additionally, this method offers a way to analyze the nanotube growth mode. In our system, we have observed an apparent base-growth mode for the lattice-oriented SWNTs. Based on the termination method and the base-growth mode, SWNTs with controlled lengths have been grown by pre-defining the relative position of the catalyst particles and the nanobarriers.

\section{Acknowledgements}

This work was supported by the National Science Foundation of China (20673004, 20725307, and 50821061) and Ministry of Science and Technology of China (2006CB932701, 2006CB932403, and 2007CB936203). C. Q. Feng acknowledges Mr. L. M. Xie (Peking University) for his useful discussion.

\section{References}

[1] lijima, S.; Ichihashi, T. Single-shell carbon nanotubes of 1-nm diameter. Nature 1993, 363, 603-605.

[2] Jiang, K. L.; Feng, C.; Liu, K.; Fan, S. S. A vapor-liquid -solid model for chemical vapor deposition growth of carbon nanotubes. J. Nanosci. Nanotechnol. 2007, 7, 1494-1504.

[3] Li, Y. M.; Kim, W.; Zhang, Y. G.; Rolandi, M.; Wang, D. W.; Dai, H. J. Growth of single-walled carbon nanotubes from discrete catalytic nanoparticles of various sizes. $J$. 
Phys. Chem. B 2001, 105, 11424-11431.

[4] Fu, Q.; Huang, S. M.; Liu, J. Chemical vapor depositions of single-walled carbon nanotubes catalyzed by uniform $\mathrm{Fe}_{2} \mathrm{O}_{3}$ nanoclusters synthesized using diblock copolymer micelles. J. Phys. Chem. B 2004, 108, 6124-6129.

[5] Bachilo, S. M.; Balzano, L.; Herrera, J. E.; Pompeo, F.; Resasco, D. E.; Weisma, R. B. Narrow $(n, m)$-distribution of single-walled carbon nanotubes grown using a solid supported catalyst. J. Am. Chem. Soc. 2003, 125, 11186-11187.

[6] Wang, B.; Poa, C. H. P.; Wei, L.; Li, L. J.; Yang, Y. H.; Chen, $Y .(n, m)$ selectivity of single-walled carbon nanotubes by different carbon precursors on Co-Mo catalysts. J. Am. Chem. Soc. 2007, 129, 9014-9019.

[7] Li, B.; Goh, C. F.; Zhou, X. Z.; Lu, G.; Tantang, H.; Chen, Y. H.; Xue, C.; Boey, F. Y. C.; Zhang, H. Patterning colloidal metal nanoparticles for controlled growth of carbon nanotubes. Adv. Mater. 2008, 20, 4873-4878.

[8] Lu, C. G.; Liu, J. Controlling the diameter of carbon nanotubes in chemical vapor deposition method by carbon feeding. J. Phys. Chem. B 2006, 110, 2025420257.

[9] Huang, S. M.; Woodson, M.; Smalley, R.; Liu, J. Growth mechanism of oriented long single walled carbon nanotubes using "fast-heating" chemical vapor deposition process. Nano Lett. 2004, 4, 1025-1028.

[10] Zheng, L. X.; O'Connell, M. J.; Doorn, S. K.; Liao, X. Z.; Zhao, Y. H.; Akhadov, E. A.; Hoffbauer, M. A.; Roop, B. J.; Jia, Q. X.; Dye, R. C.; Peterson, D. E.; Huang, S. M.; Liu, J.; Zhu, Y. T. Ultralong single-wall carbon nanotubes. Nat. Mater. 2004, 3, 673-676.

[11] Han, S.; Liu, X. L.; Zhou, C. W. Template-free directional growth of single-walled carbon nanotubes on a- and r-plane sapphire. J. Am. Chem. Soc. 2005, 127, 52945295.

[12] Ismach, A.; Segev, L.; Wachtel, E.; Joselevich, E. Atomicstep-templated formation of single wall carbon nanotube patterns. Angew. Chem. Int. Ed. 2004, 43, 6140-6143.

[13] Kocabas, C.; Hur, S. H.; Gaur, A.; Meitl, M. A.; Shim, M.; Rogers, J. A. Guided growth of large-scale, horizontally aligned arrays of single-walled carbon nanotubes and their use in thin-film transistors. Small 2005, 1, 11101116.

[14] Kang, S. J.; Kocabas, C.; Ozel, T.; Shim, M.; Pimparkar, N.; Alam, M. A.; Rotkin, S. V.; Rogers, J. A. Highperformance electronics using dense, perfectly aligned arrays of single-walled carbon nanotubes. Nat. Nanotechol. 2007, 2, 230-236.

[15] Jin, Z.; Chu, H. B.; Wang, J. Y.; Hong, J. X.; Tan, W. C.; $L i, Y$. Ultralow feeding gas flow guiding growth of largescale horizontally aligned single-walled carbon nanotube arrays. Nano Lett. 2007, 7, 2073-2079.

[16] Duan, X. J.; Zhang, J.; Ling, X.; Liu, Z. F. Nano-welding by scanning probe microscope. J. Am. Chem. Soc. 2005, 127, 8268-8269.

[17] Kocabas, C.; Kang, S. J.; Ozel, T.; Shim, M.; Rogers, J. A. Improved synthesis of aligned arrays of single-walled carbon nanotubes and their implementation in thin film type transistors. J. Phys. Chem. C 2007, 111, 1787917886.

[18] Ago, H.; Ishigami, N.; Yoshihara, N.; Imamoto, K.; Akita, S.; Ikeda, K.; Tsuji, M.; Ikuta, T.; Takahashi, K. Visualization of horizontally-aligned single-walled carbon nanotube growth with ${ }^{13} \mathrm{C} /{ }^{12} \mathrm{C}$ isotopes. J. Phys. Chem. C 2008, 112, 1735-1738.

[19] Ding, L.; Yuan, D. N.; Liu, J. Growth of high-density parallel arrays of long single-walled carbon nanotubes on quartz substrates. J. Am. Chem. Soc. 2008, 130, 54285429.

[20] Liu, X. L.; Ryu, K.; Badmaev, A.; Han, S.; Zhou, C. W. Diameter dependence of aligned growth of carbon nanotubes on a-plane sapphire substrates. J. Phys. Chem. C 2008, 112, 15929-15933.

[21] Geblinger, N.; Ismach, A.; Joselevich, E. Self-organized nanotube serpentines. Nat. Nanotechol. 2008, 3, 195200.

[22] Yuan, D. N.; Ding, L.; Chu, H. B.; Feng, Y. Y.; McNicholas, T. P.; Liu, J. Horizontally aligned single-walled carbon nanotube on quartz from a large variety of metal catalysts. Nano Lett. 2008, 8, 2576-2579. 